\title{
Parásitos y Lesiones Histopatológicas en Branquias de Gamitanas (Colossoma macropomum) Juveniles bajo Crianza Semiintensiva
}

\author{
Parasites and Histopathological Lesions in Gills of Juvenile Gamitana \\ (Colossoma macropomum) RAISED IN SEMI-INTENSIVE FARMING
}

\author{
Mario Vargas L. ${ }^{1,4}$, Nieves Sandoval C. ${ }^{1}$, Eva Casas A. ${ }^{2}$, Gloria Pizango P. ${ }^{3}$, \\ Alberto Manchego S. ${ }^{2}$
}

\section{RESUMEN}

\begin{abstract}
El objetivo del presente trabajo fue determinar el tipo y frecuencia de parásitos en branquias de gamitana (Colossoma macropomum) y describir las lesiones histopatológicas asociadas a su presencia. Se trabajó con 30 gamitanas juveniles, tomadas al azar de una piscigranja en Iquitos, Perú. Los arcos branquiales del lado izquierdo fueron colocados en placas Petri con agua destilada para la observación de la presencia de parásitos y los del lado derecho se fijaron en formol al 5\% para el estudio histopatológico. Asimismo, se determinó la calidad del agua del estanque. Se identificaron tres tipos de ectoparásitos: monogeneos, pertenecientes a la familia Dactylogyridae, Subfamilias Anacanthorinae y Ancyrocephalinae (100\%, 30/30), una especie de protozoo de la Familia Oodinidae, género Piscinoodinium $(36.7 \%, 11 / 30)$ y un artrópodo de la Clase Maxillopoda, Subclase Copepoda (20\%, 6/30). Dentro de las lesiones histopatológicas se observaron trastornos inflamatorios como presencia de células granulares eosinofílicas (100\%) y trastornos de adaptación como hiperplasia del epitelio (96.7\%), fusión lamelar (80\%) y atrofia lamelar (60\%). Asimismo, el oxígeno disuelto estaba en niveles cercanos a los letales, el pH correspondía a un medio ácido y los valores de $\mathrm{CO}_{2}$ se encontraban fuera de los rangos esperados.
\end{abstract}

Palabras clave: gamitana, Colossoma macropomum, monogeneo, Piscinoodinium, copépodo, ectoparásitos branquiales

\footnotetext{
${ }^{1}$ Laboratorio de Histología, Embriología y Patología Veterinaria, ${ }^{2}$ Laboratorio de Microbiología y Parasitología Veterinaria, Facultad de Medicina Veterinaria, Universidad Nacional Mayor de San Marcos, Lima, Perú

${ }^{3}$ Departamento Académico de Hidrobiología, Facultad de Ciencias Biológicas, Universidad Nacional de la Amazonía Peruana, Iquitos, Perú

${ }^{4}$ E-mail: vargasleyva324@gmail.com
}

Recibido: 18 de abril de 2015

Aceptado para publicación: 14 de julio de 2015 
The aim of this study was to determine the type and frequency of parasites in gamitana gills (Colossoma macropomum) and to describe the associated histopathological lesions. Thirty specimens were taken from a fish farm in Iquitos, Peru. The left branchial arches were placed in Petri dishes with distilled water to observe the presence of parasites and the right arches were fixed with formalin 5\% for histopathological studies. Besides, water quality of the pond was analyzed. Three types of ectoparasites were identified: monogeneans, members of the Family Dactylogyridae, Subfamilies Anacanthorinae and Ancyrocephalinae (100\%, 30/30), ones protozoan, member of the Family Oodinidae, genus Piscinoodinium $(36.7 \%, 11 / 30)$, and one arthropod, member of the Class Maxillopoda, Sub-class Copepoda (20\%, 6/30). Among the histopathological lesions, the most common were inflammatory disorders: presence of eosinophilic granule cells (100\%), and adaptation disorders: epithelial hyperplasia (96.7\%), lamellar fusion (80\%) and lamellar atrophy (60\%). Moreover, dissolved oxygen was close to lethal levels, $\mathrm{pH}$ was acid and $\mathrm{CO}_{2}$ were outside the expected range.

Key words: gamitana, Colossoma macropomum, monogenean, Piscinoodinium, copepod, gill ectoparasites

\section{INTRODUCCIÓN}

La ictiofauna de la Amazonia está representada por más de 2000 especies de peces, de las cuales, cerca de 800 viven en las aguas de los ríos peruanos (Ortega et al., 2011). El potencial pesquero de los ríos amazónicos es inmenso; sin embargo, poco esfuerzo se realiza para el manejo racional del recurso y, lo que es peor, la deforestación y la contaminación de las aguas, aunadas al desarrollo de las ciudades y asentamientos humanos acostumbrados al consumo de pescado han puesto en peligro esta diversidad y, con ello, la fuente de alimento del que dependen los pobladores de la región.

Por esta razón, la piscicultura es una de las grandes alternativas con posibilidades de desarrollo para la región amazónica, no solo por la gran abundancia de espejos y cursos de agua, sino también por las especies nativas promisorias. Una de las especies importantes para cultivo es la gamitana (Colossoma macropomum), uno de los peces de escama más grandes de la cuenca amazónica, que puede llegar hasta $90 \mathrm{~cm}$ de longitud total y con peso de $30 \mathrm{~kg}$ (Goulding, 1980).

El cuerpo de la gamitana es comprimido, de coloración gris oscuro en el dorso y amarillo blancuzco en la parte ventral; patrón de coloración que puede variar en función del tipo de agua donde se desarrolla (Guerra, 1999). Se encuentra en los ríos de Brasil, Colombia, Venezuela y Perú y ha sido introducida en países centroamericanos y asiáticos.

Los peces dulceacuícolas, tanto en condiciones naturales como de cultivo, son susceptibles al ataque de agentes patógenos facultativos, que usualmente no afectan su desarrollo ni estado de salud, ya que son reducidos por las defensas del organismo; sin embargo, si las condiciones se tornan desfavorables para los peces (cambio brusco de temperatura, deficiencia de oxígeno, alta densidad de peces, elementos tóxicos en el agua), sus defensas naturales pueden mermar y el organismo atacante invade al hospedero, convirtiéndose en un agente patógeno, ocasio- 
Cuadro 1. Valores de parámetros fisicoquímicos del agua en cultivo semiintensivo de juveniles Colossoma macropomum en la Piscigranja Quistococha, FCB UNAP (Junio, 2006)

\begin{tabular}{lcc}
\hline Parámetro & Valores esperados & Valores de los estanques \\
\hline Oxígeno disuelto $(\mathrm{mg} / \mathrm{L})$ & $6.0-7.0$ & 2.19 \\
$\mathrm{pH}$ & $7.0-8.0$ & 6 \\
Temperatura $\left({ }^{\circ} \mathrm{C}\right)$ & $24-28$ & 27.6 \\
$\mathrm{CO}_{2}(\mathrm{mg} / \mathrm{L})$ & $1.8-2.0$ & 2.8 \\
Alcalinidad $(\mathrm{mg} / \mathrm{L})$ & $30-200$ & 40 \\
\hline
\end{tabular}

${ }^{1}$ Fuente: W oynarovich A y Woynarovich E (1998); FAO (1989)

nando altas tasas de mortalidad en la piscigranjas.

El uso de la gamitana como pez de cultivo en piscigranjas se ha desarrollado notablemente en la región, pero con ello se ha podido detectar la aparición de agentes patógenos que pueden afectar la salud de los peces, especialmente parásitos, mermando la producción y ocasionando pérdidas económicas (Centeno et al., 2004). No se han realizado estudios en el Perú sobre la fauna parasitaria de la gamitana en cultivo; por ello el objetivo del presente estudio fue identificar la presencia y frecuencia de parásitos en las branquias de gamitana, así como determinar las lesiones histopatológicas asociadas a su presencia.

\section{MATERIALES Y MéTODos}

\section{Lugar de Estudio}

El muestreo de los peces fue realizado en la piscigranja Quistococha, del Centro de Investigación, Experimentación y Enseñanza de la Facultad de Ciencias Biológicas (FCB), Universidad Nacional de la Amazonía Peruana (UNAP). El centro experimental está ubicado en la Carretera Iquitos-Nauta km 6, margen izquierda, distrito de San Juan Bautista, provincia de Maynas, región Loreto. El análisis de las lesiones histopatológicas se realizó en la Sección de Ictiopatología de la Facultad de Medicina Veterinaria de la Universidad Nacional Mayor de San Marcos, Lima.

Los parámetros fisicoquímicos fueron medidos utilizando el LaMotte Fresh Water Aquaculture Outfit Model AQ-3 Kit. Los parámetros del agua se muestran en el Cuadro 1. El oxígeno disuelto estaba en niveles cercanos a los letales, el $\mathrm{pH}$ correspondía a un medio ácido y los valores de $\mathrm{CO}_{2}$ se encontraban fuera de los rangos esperados.

\section{Peces y Muestras}

Se colectaron 30 gamitanas al azar, en un lapso de 30 días. Los peces fueron trasladados vivos en bolsas de polietileno con agua hasta el laboratorio del Departamento Académico de Hidrobiología de la FCB-UNAP. Los peces fueron sacrificados con un estilete, haciendo un corte medular, previa sedación con isoeugenol.

Los especímenes utilizados tuvieron un peso promedio de $320 \mathrm{~g}$ y una longitud total de $24.9 \mathrm{~cm}$. El peso se obtuvo en una balanza Ohaus de $\pm 1 \mathrm{~g}$ de precisión y la longitud se midió con un ictiómetro graduado en centímetros.

En la necropsia se seccionaron los opérculos y se extrajeron los cuatro arcos- 
Cuadro 2. Especies de ectoparásitos identificadas en 30 gamitanas juveniles (Colossoma macropomum) de cultivo semiintensivo en la FCB-UNAP (2006)

\begin{tabular}{lc}
\hline Ectoparásitos branquiales & $\%$ \\
\hline Monogeneo & 100.0 \\
Protozoo & 36.7 \\
Copépodo & 20.0 \\
Monogeneo y copépodo & 16.7 \\
Monogeneo y protozoo & 33.3 \\
Monogeneo, copépodo y & 3.3 \\
protozoo & \\
\hline
\end{tabular}

branquiales. Los del lado izquierdo se colocaron en placas Petri con agua destilada para la observación de parásitos (estereoscopio Zeiss) y los del lado derecho fueron conservados en formol al 5\%, para la identificación de las lesiones.

\section{Evaluación de Parásitos}

Los monogeneos y copépodos fueron coloreados mediante la técnica de Malmberg. Para la identificación de monogeneos se observaron las estructuras esclerotizadas del haptor y del complejo copulatorio y se utilizó la clave para monogeneos neotropicales de aguas dulces descrita por Thatcher (1991). Para el caso de los copépodos se utilizaron las características descritas por Thatcher y Boeger (1983), y para la identificación del protozoo Piscinoodinium sp se emplearon las descripciones indicadas por Centeno et al. (2004).

\section{Estudio Histopatológico}

Se utilizaron técnicas histopatológicas para identificar a los ectoparásitos branquiales, así como para identificar las alteraciones histopatológicas ocasionadas por estos parásitos (Eiras et al., 2000). El filamento branquial fue observado considerando tres partes (ápice, medio y base) y así poder clasificar las lesiones de acuerdo a la ubicación dentro del filamento branquial.

\section{Análisis de Datos}

Los cambios y alteraciones histopatológicas en las branquias fueron clasificados como trastornos inflamatorios, circulatorios, del crecimiento y degenerativos.

La frecuencia de las alteraciones en las branquias fue clasificada por su extensión como:

- Escasa: de $0 \%$ hasta $25 \%$ de la superficie.

- Leve: más del $25 \%$ hasta $50 \%$ de la superficie.

- Moderada: más del 50\% hasta 75\% de la superficie.

Se empleó como método estadístico la prueba de diferencia de proporciones para determinar si existía asociación entre la ubicación del parásito y las lesiones presentadas en branquias (ápice, medio y base).

\section{Resultados}

\section{Observación Macroscópica}

La mayoría de las branquias al momento de la inspección ocular presentaba un color rojizo, de forma y tamaño aparentemente normal. El $100 \%$ se encontraba parasitado (Cuadro 2), donde el 46.7\% (14/30) estaba infectado por un solo tipo de parásito, en tanto que el $53.3 \%(16 / 30)$ estaba infectado por dos o más tipos de parásitos.

Se identificaron tres tipos de ectoparásitos: monogeneos, pertenecientes a la Familia Dactylogyridae, Subfamilia Anacanthorinae (100\%) y Subfamilia Ancyrocephalinae (100\%) (Fig. 1); un tipo de protozoario perteneciente a la Familia Oodinidae, género Piscinoodinium y un artrópodo de la Clase Maxillopoda, Sub-clase Copépoda (Fig. 2). Los parásitos se distribuían por toda la branquia. 


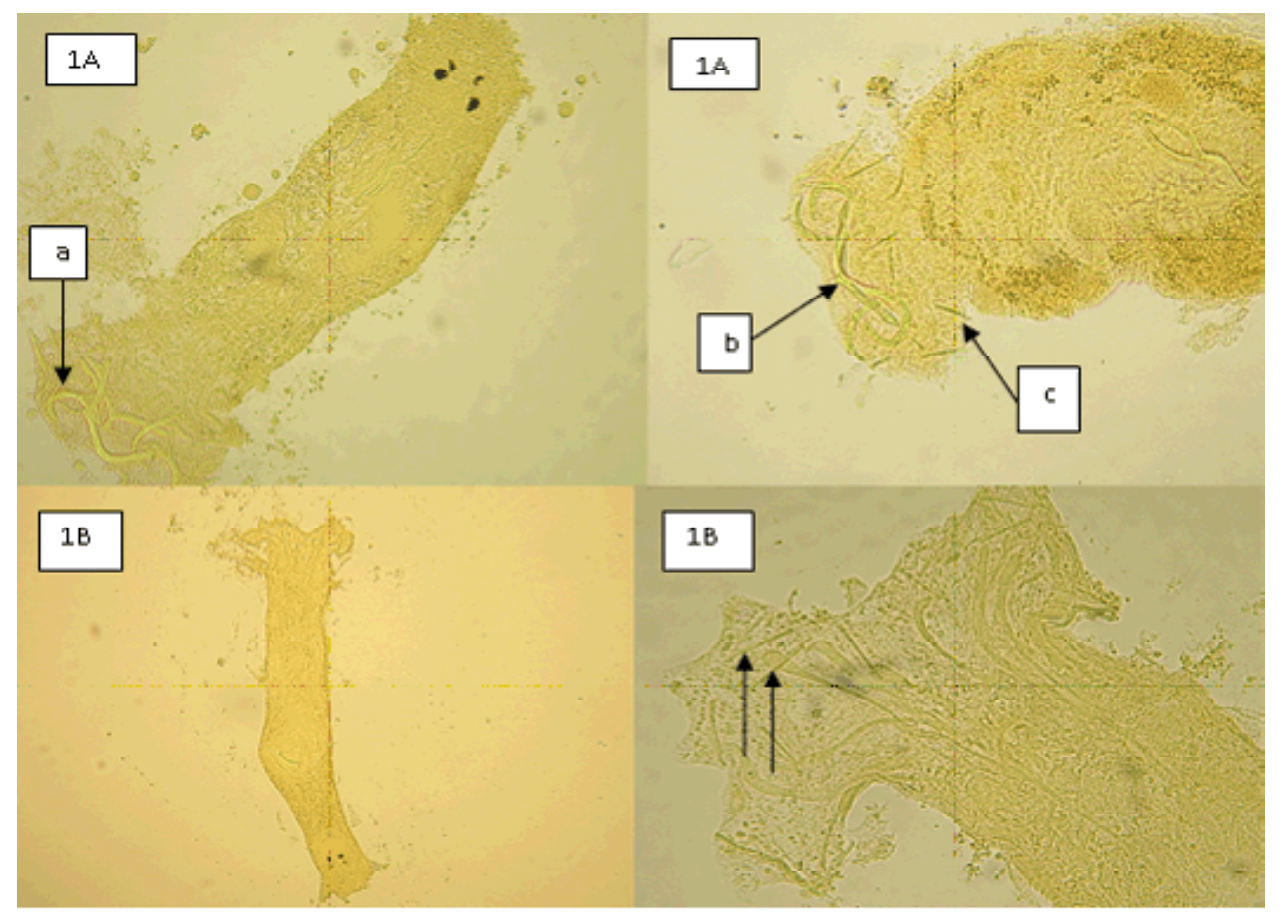

Figura 1. Ectoparásitos branquiales. (A) Monogeneo de la Subfamilia Ancyrocephalinae. Se observa el opistohaptor con 2 pares de macroganchos (a), 2 barras (b) y 14 microganchos (c). (B) Monogeneo de la Subfamilia Anacanthorinae, donde el opistohaptor carece de macroganchos y barras, presentando solo 16 a 18 microganchos (flechas) (Técnica de Malmberg)

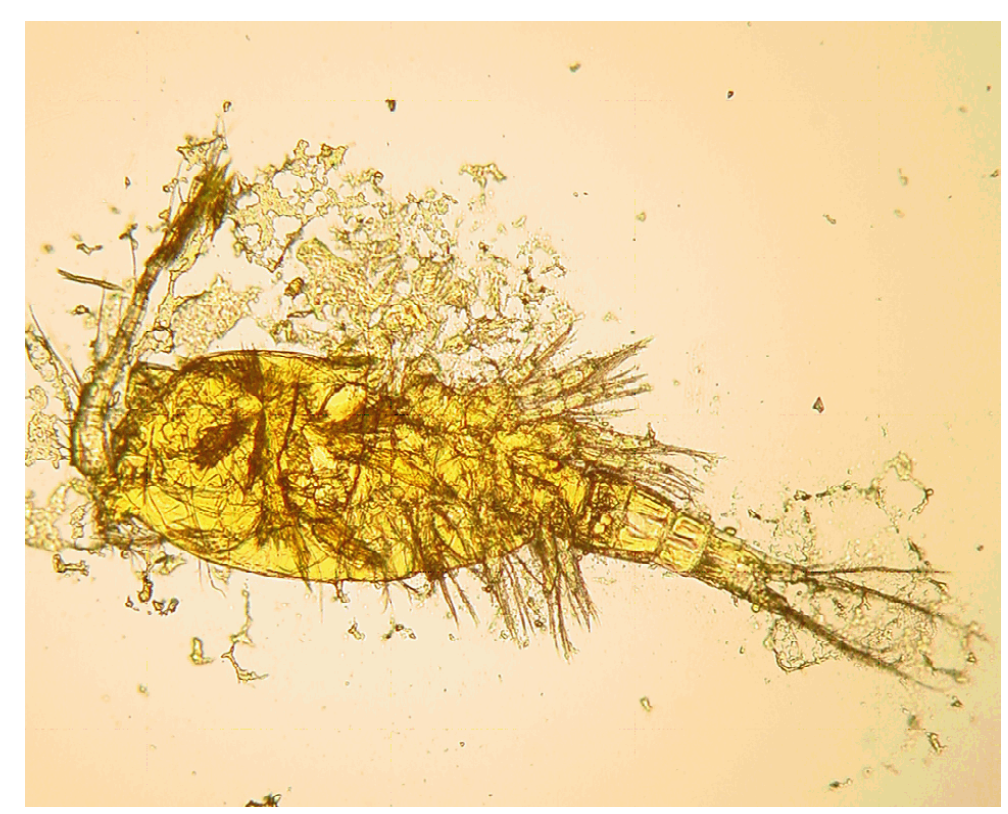

Figura 2. Ectoparásito branquial, copépodo. Se observa el tegumento recubierto de quitina, a veces calcificada, con apéndices articulados, cabeza, tórax y abdomen (Técnica de Malmberg, 400x) 


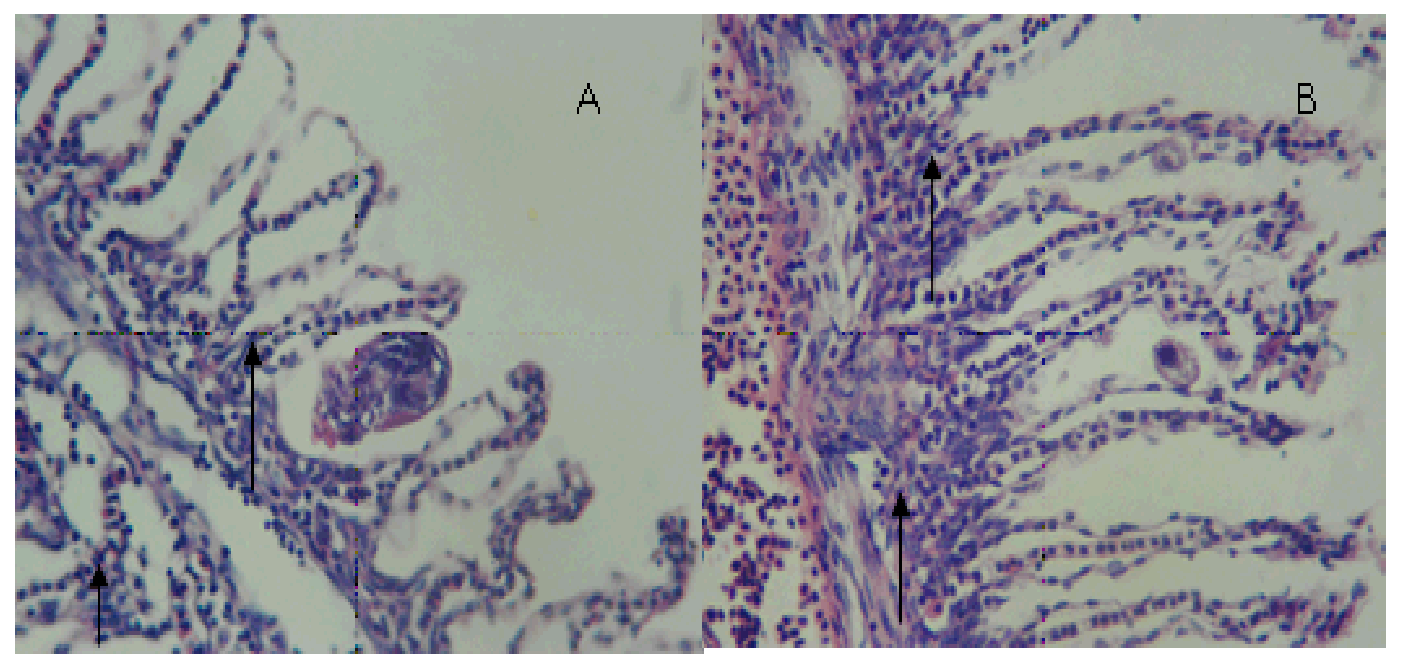

Figura 3. Ectoparásitos branquiales. (A) Monogeneo causando atrofia lamelar y necrosis de la lamela (flechas). (B) Piscinoodinium sp. Presencia de células granulares eosinofilicas e hiperplasia del epitelio (flechas). H-E, 400x

La ubicación de los monogeneos en el filamento fue muy característica, y se visualizó como un evento repetitivo. Los más grandes $(0.7 \mathrm{~mm})$ se ubicaban en la parte media del filamento y los más pequeños $(0.35 \mathrm{~mm})$ hacia los extremos (ápice y base). El opistohaptor de los monogeneos ubicados en la parte media carecía de anclas y barras, y presentaba de 16 a 18 microganchos, característica que permite determinar que pertenecían a la subfamilia Anacanthorinae. Asimismo, los monogenos cuyos extremos de los filamentos branquiales presentaban el opistohaptor con 2 pares de anclas, 2 barras y 14 microganchos eran características morfológicas que identifican a la subfamilia Ancyrocephalinae.

Se identificaron, además, otras estructuras en la superficie de la branquia. Tenían forma circular u ovalada, inmóviles, a manera de puntos de color blanquecino, midiendo $162 \mu \mathrm{m}$ de diámetro, que fueron identificadas como el dinoflagelado Piscinoodinium sp. Fueron encontrados en el $36.7 \%$ (11/30) de los peces, donde seis de ellos se distribuían en la parte media y cinco en la base del filamento de la branquia. Por otro lado, los copépodos se encontraron en la parte media del filamento branquial (6/30).

\section{Observación Histopatológica}

En la evaluación macroscópica se encontró la presencia de parásitos en todas las branquias; sin embargo, en la evaluación histopatológica, solo el 33.3\% (10/30) de las branquias estaban parasitadas por un género de parásito y en el $66.7 \%$ (20/30) por dos géneros, trematodos monogenésicos (22/30) y Piscinoodinium sp (28/30).

Piscinoodinium sp se ubicó entre dos filamentos branquiales y en el espacio interlamelar. Estos se encontraron tanto en la parte media del filamento branquial (24/28), como en la base (22/28) y en el ápice (19/28). Los monogeneos se ubicaron mayormente en el ápice (16/22), seguido de la base $(12 / 22)$ y parte media $(11 / 22)$.

En los filamentos branquiales se observaron células granulares eosinofílicas, hiperemia, hiperplasia del epitelio, atrofia lamelar, fusión lamelar, necrosis lamelar, indicando branquitis (figuras 3 y 4 ). 

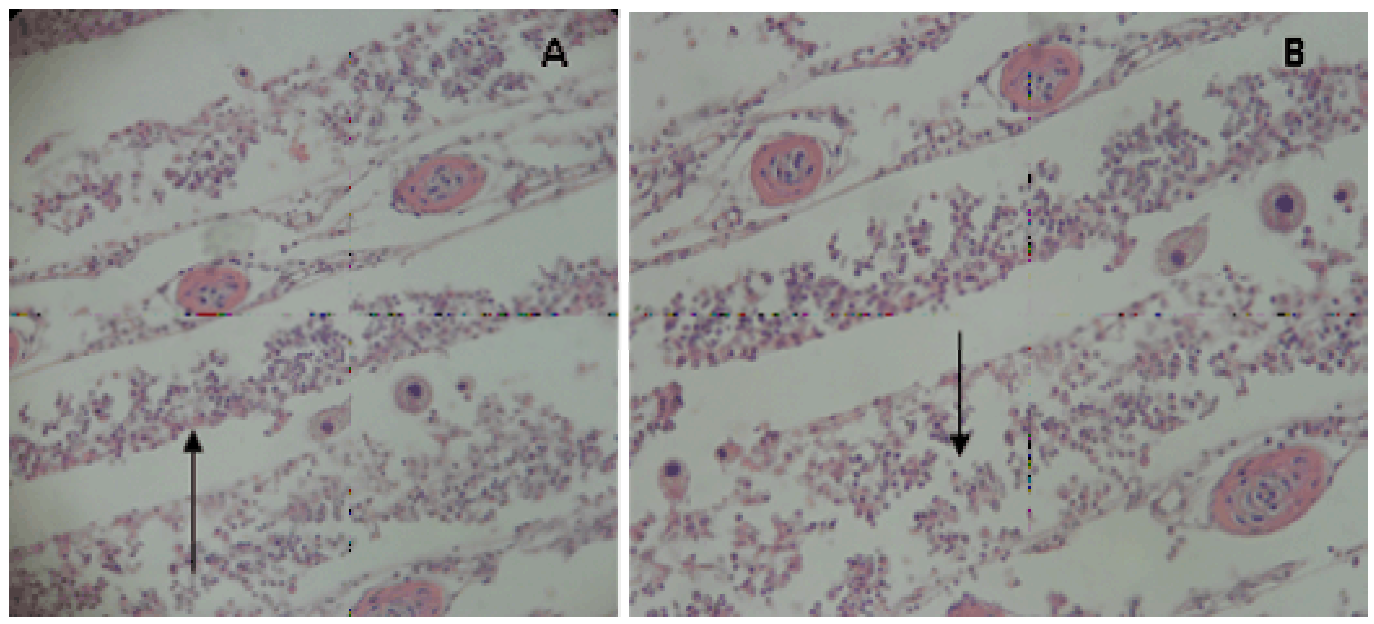

Figura 4. Ectoparásitos branquiales. (A) Piscinodinium sp causando atrofia lamelar y fusión lamelar (flecha). (B) Piscinoodinium sp causando necrosis lamelar (flecha). H-E, 400x

Cuadro 3. Tipo y distribución de lesiones microscópicas en el filamento branquial de 30 gamitanas juveniles (Colossoma macropomum) en cultivo semiintensivo en la FCB-UNAP (2006)

\begin{tabular}{|c|c|c|c|c|c|c|c|}
\hline \multirow{2}{*}{\multicolumn{2}{|c|}{ Trastornos en la branquia }} & \multicolumn{6}{|c|}{ Partes del filamento branquial } \\
\hline & & \multicolumn{2}{|c|}{ Ápice } & \multicolumn{2}{|c|}{ Medio } & \multicolumn{2}{|c|}{ Base } \\
\hline & & $\mathrm{N}^{\mathrm{o}}$ & $\%$ & $\mathrm{~N}^{\mathrm{o}}$ & $\%$ & $\mathrm{~N}^{\mathrm{o}}$ & $\%$ \\
\hline Inflamatorios & $\begin{array}{l}\text { Células granulares } \\
\text { eosinofílicas }\end{array}$ & 11 & 36.7 & 17 & 56.7 & 15 & 50.0 \\
\hline Circulatorios & Hiperemia & 10 & 33.3 & 12 & 40.0 & 7 & 23.3 \\
\hline \multirow[t]{3}{*}{ De adaptación } & $\begin{array}{l}\text { Hiperplasia del } \\
\text { epitelio }\end{array}$ & 18 & 60.0 & 20 & 16.0 & 16 & 53.3 \\
\hline & Atrofia lamelar & 9 & 30.0 & 12 & 40.0 & 7 & 23.3 \\
\hline & Fusión lamelar & 16 & 53.3 & 17 & 56.7 & 10 & 33.3 \\
\hline Degenerativos & Necrosis lamelar & 3 & 10.0 & 3 & 10.0 & 1 & 3.3 \\
\hline
\end{tabular}

La distribución de las lesiones en el filamento branquial se presentó mayormente en la parte media del filamento, relacionado a los trastornos inflamatorios y a los trastornos de adaptación; este último asociado a hiperplasia del epitelio (Cuadro 3). No obstante, la prueba de diferencia de proporciones mostró que no existe asociación entre la ubicación del parásito y las lesiones presentadas.

La extensión de las alteraciones en la branquia fue de grado diverso, predominando el grado severo en trastornos inflamatorios (16/30) y de adaptación asociados a la hiperplasia del epitelio (16/30) (Cuadro 4). 
Cuadro 4. Grado de lesiones histopatológicas en el arco branquial de 30 gamitanas juveniles (Colossoma macropomum) en cultivo semiintensivo en la FCB-UNAP (2006)

\begin{tabular}{lccccc}
\hline Trastornos & Escaso & Leve & Moderado & Severo & $\%$ \\
\hline $\begin{array}{l}\text { Inflamatorios } \\
\quad \text { Células granulares }\end{array}$ & 0 & 7 & 7 & 16 & 100.0 \\
$\quad \begin{array}{c}\text { eosinofílicas } \\
\text { Circulatorios }\end{array} \quad$ & & & & & \\
$\quad$ Hiperemia & 0 & 2 & 5 & 5 & 40.0 \\
De adaptación & & & & & \\
$\quad$ Hiperplasia del epitelio & 0 & 6 & 7 & 16 & 96.7 \\
$\quad$ Atrofia lamelar & 0 & 4 & 7 & 7 & 60.0 \\
$\quad$ Fusión lamelar & 0 & 4 & 7 & 13 & 80.0 \\
Degenerativos & & & & & \\
$\quad$ Necrosis lamelar & 0 & 3 & 1 & 1 & 16.7 \\
\hline
\end{tabular}

\section{Discusión}

No se evidenciaron signos clínicos de un pez enfermo al momento de la colecta, lo que estaría relacionado a la baja carga parasitaria encontrada; sin embargo, aún no se han determinado las cargas parasitarias causantes de enfermedad en la gamitana. Iregui et al. (1999) mencionan que la presencia de un agente potencialmente patógeno en un hospedador no es sinónimo de enfermedad.

La elevada infestación de monogeneos encontrada en el presente estudio fue similar a los hallazgos de Centeno et al. (2004) en Colossoma macropomum y en el híbrido $C$. macropomum x Piaractus brachypomus; donde, además, reportaron al monogeneo Anacanthorus spatulatus como la especie más predominante $(96.4 \%)$. De la misma manera, otros investigadores señalan a los monogeos como los parásitos de mayor frecuencia (Aragort, 1994; Verján et al., 2001; Martins et al., 2002; Flores J y Flores R, 2003), siendo un peligro potencial para el cul- tivo de peces en aguas tropicales y semitropicales. Asimismo, Santos et al. (2013) reportaron que las mayores tasas de infestación en gamitanas fueron por protozoos, seguidos de monogeneos (96.7 y $95 \%$, respectivamente).

En el presente estudio se encontraron infestaciones mixtas con Anacanthorus sp y Ancyrocephalinae. Aragort et al. (2002), asimismo, reportaron infecciones mixtas de monogeneos (Anacanthorus spatulatus y Linguadactyloides brinkmani) en peces $C$. macropomum en cultivo.

La frecuencia de Piscinoodinium sp (28/30 en la evaluación histopatológica) fue bastante más alta al $18 \%$ mencionado por Verján et al. (2001) en branquias de cachama blanca (Piaractus brachypomus) en dos piscigranjas; no obstante, Martins et al. (2002) reportaron niveles similares de infección por Piscinoodium pillulare en peces de agua fresca cultivados en el estado de Sao Paulo, Brasil. Santos et al. (2013) también pudieron identificar este parásito en branquias en el 
97\% de los especímenes. La alta frecuencia de Piscinoodinium sp podría deberse al modelo de distribución de pozas tipo rosario, que favorecería la trasmisión del parásito.

En el caso de los copépodos, la infestación encontrada en el presente estudio fue baja e inferior al 10.5\% de Ergasilus sp reportado por Ardaya et al. (1996) en branquias de cachamas cultivadas en Bolivia. Por otro lado, en un estudio en Venezuela, no se encontró Ergasilus sp en Colossoma macropomum (Centeno et al., 2004). Los ergasilideos parasitan peces de los órdenes Clupeiformes, Cypriniformes, Siluriformes, Atheriniformes y Perciformes en peces de la amazonia brasilera (Thatcher et al., 1983), afectando no solo las branquias sino, además, pueden localizarse en piel y fosas nasales (Thatcher, 1998).

Los trastornos del crecimiento en las branquias, tales como la hiperplasia del epitelio (29/30) y atrofia lamelar (18/30), así como fusión lamelar (18/30), fueron hallazgos comunes en el presente estudio. Noga (2010) afirma que los trastornos del crecimiento son las respuestas más frecuentes ante infecciones por bacterias y parásitos y por una pobre calidad de agua.

Verjan et al. (2001) determinaron una asociación entre el $\mathrm{pH}$ del agua con la pérdida de lamelas, la presencia de trematodos y mixosporidios y la necrosis del epitelio lamelar. En ese estudio se encontró que valores menores de 6 y mayores de 8 fueron factores de riesgo para la pérdida de lamelas y valores menores de 6 con necrosis epitelial. Asimismo, los nitritos, amonio y turbidez se asociaron con necrosis epitelial e hipertrofia del epitelio lamelar. Dichos resultados demuestran que otros factores, además de los parásitos, pueden afectar la salud de las branquias. El sistema de cultivo de la granja del presente estudio es de tipo rosario, lo que genera que el agua circule por diversas pozas y llegue agua de mala calidad a las pozas finales del cultivo. Los peces muestreados procedieron de uno de los estanques de la parte baja. Es así que la calidad de agua del estanque podría haber sido un factor adicional, en el presente estudio, que afectó el estado de las branquias.

\section{Conclusiones}

- Se identificaron tres tipos de ectoparásitos en las branquias de gamitanas: a) monogeneos pertenecientes a la Familia Dactylogyridae, Subfamilias Anacanthorinae y Ancyrocephalinae, en el 100\% de los peces, b) un tipo de protozoario perteneciente a la Familia Oodinidae, Género Piscinoodinium en el $36.7 \%$ de los peces, y c) un artrópodo de la Clase Maxillopoda, Subclase Copépoda en el $20 \%$ de los peces.

- Las lesiones histopatológicas en branquias que se evidenciaron con mayor frecuencia fueron trastornos inflamatorios (células granulares eosinofilicas) (100\%), hiperplasia del epitelio (96.7\%), fusión lamelar (80\%) y atrofia lamelar $(60 \%)$.

- La pobre calidad del agua de los estanques de cultivo tendrían un rol importante en el establecimiento de la infestación $\mathrm{y}$ en desarrollo de lesiones branquiales.

\section{Literatura Citada}

1. Aragort W. 1994. Parasitismo por tremátodos monogenésicos branquiales en cachamas, Colossoma macropomum bajo condiciones de cultivo. Tesis de Maestría. Maracay, Venezuela: Universidad Central de Venezuela (UCV). 107 p.

2. Aragort W, Morales $G$ León $E$, Pino AL, Guillén A, Silva M. 2002. Patologías asociadas a monogeneos branquiales en cachama bajo cultivo. Veterinaria Trop 27(2): 75-85.

3. Ardaya D, Castedo C, Cerruto A, Conroy D, Corcuy A, Cuellar M, Morales $G$. 1996. Evaluación preliminar de 
la presencia de algunas enfermedades y parásitos en el cultivo de cachama (Colossoma macropomum) y pacus (Piaractus mitrei) en Santa Cruz, Bolivia. En: Memorias III Congreso de Ciencias Veterinarias. Maracay, Venezuela.

4. Centeno L, Silva-Acuña A, Silva-Acuña R, Pérez JL. 2004. Fauna ectoparasitaria asociada a Colosssoma macropomun y al híbrido de Colossoma macropomun x Piaractus brachypomus, cultivados en el estado Delta Amacuro, Venezuela. Bioagro 16: 121-126.

5. Eiras JC, Takemoto RM, Pavanelli GC. 2000. Métodos de estudio y técnicas laboratoriales en parasicología de peces. Zaragoza, España: Ed Acribia. 133 p.

6. [FAO] Organización de las Naciones Unidas para la Agricultura y la Alimentación. 1989. Avances en el cultivo de peces del genero Colossoma. Documento de Campo N ${ }^{\circ}$ 5. Roma: FAO. 235 p.

7. Flores J, Flores R. 2003. Monogeneos, parásitos de peces en México: estudio recapitulativo. Rev Téc Pec Méx 41: 175-192.

8. Goulding M. 1980. The fishes and the forest. Explorations in Amazonian natural history. London: University of California Press. 280 p.

9. Guerra H. 1999. Piscicultura en la Amazonía peruana. Iquitos, Perú: IICA. $59 \mathrm{p}$.

10. Iregui $C$, Eslava P, Martinez E, Figueroa J. 1999. Descripción de un caso de mixosporidiasis clinica en cachama blanca, Piaractus brachypomus. Dhalia 3: 17-29.

11. Martins ML, Onaka EM, Ruas De Moraes F, Bozzo FR, De Mello A, Paiva FC, Goncalves A. 2002. Recent studies on parasitic infections of freshwater cultivated fish in the state of Sao Paulo, Brazil. Acta Scientiarum 24: 981- 985.

12. Noga J. 2010. Fish disease. Diagnosis and treatment. $2^{\text {nd }}$ ed. USA: Iowa State University Press. 519 p.
13. Ortega H, Hidalgo M, Trevejo G, Correa E, Cortijo A, Meza V, Espino J. 2011. Lista anotada de los peces de aguas continentales del Perú: estado actual del conocimiento, distribución, usos y aspectos de conservación. Lima: Ministerio del Ambiente. Dirección General de Diversidad Biologica. 58 p.

14. Santos EF, Tavares-Dias M, Pinheiro DA, Neves LR, Barbosa Marinho RG, Dias MKR. 2013. Fauna parasitária de tambaqui Colossoma macropomum (Characidae) cultivado em tanque-rede no estado do Amapá, Amazônia oriental. Acta Amaz 43: 105-112. doi: 10.1590/ S0044-59672013000100013

15. Thatcher VE, Boeger WA. 1983. Patología de peixes da amazonia brasileira. 3. Alteracoes histológicas em branquias provocadas por Ergasilus, Brasergasilus e Acusicola (Crustacea: Cyclopoida: Ergasilidae). Acta Amaz 13: 441-451.

16. Thatcher VE. 1991. Amazon fish parasites. En: Aquatic biodiversity in Latin America. Vol 1. Bulgaria: Pensoft Publishers. $393 \mathrm{p}$.

17. Thatcher VE. 1998. Copepods and fishes in the Brazilian Amazon. J Marine Syst 15: 97-112. doi: 10.1016/S09247963(97)00043-2

18. Verjan N, Uregui CA, Rey AL, Donado $P$. 2001. Sistematización y caracterización de las lesiones branquiales de la cachama blanca (Piaractus brachypomus) de cultivo clínicamente sana: algunas interacciones hospedador - patógeno - ambiente. Aquatic 15. [Internet]. Disponible en: http:// www.revistaaquatic.com/aquatic/ art.asp? $\mathrm{t}=\mathrm{h} \& \mathrm{c}=132$

19. Woynarovich A, Woynarovich E. 1998. Reproducción artificial de las especies Colossoma y Piaractus. Una guía detallada de la producción de los alevinos de gamitana, paco y caraña. FONDEPES. Fondo Nacional de Desarrollo Pesquero. $66 \mathrm{p}$. 